\title{
Astrocyte gp I30-expression is critical for the control of Toxoplasma encephalitis
}

\author{
Katrin Drögemüller ${ }^{1}$, Ulrike Helmuth ${ }^{1}$, Anna Brunn², Monika Sakowicz- \\ Burkiewicz ${ }^{1,2}$, Dirk Reinhold ${ }^{3}$, David H Gutmann" ${ }^{4}$, Werner Mueller ${ }^{5,6}$, \\ Martina Deckert ${ }^{2}$ and Dirk Schlüter*1
}

\author{
Address: ${ }^{1}$ Institut für Medizinische Mikrobiologie, OvG Universität Magdeburg, 39120 Magdeburg, Germany, ${ }^{2}$ Abteilung für Neuropathologie, \\ Universität zu Köln, 50937 Köln, Germany, ${ }^{3}$ Institut für Immunologie, OvG Universität Magdeburg, 39120 Magdeburg, Germany, ${ }^{4}$ Department \\ of Neurology, Washington University School of Medicine, St. Louis, MO 63110, USA, ${ }^{5}$ Helmholtz-Zentrum für Infektionsforschung, 38124 \\ Braunschweig, Germany and ' ${ }^{2}$ niversity of Manchester, Manchester, UK \\ Email: Dirk Schlüter* - dirk.schlueter@med.ovgu.de \\ * Corresponding author
}

from Infectious diseases of the nervous system: pathogenesis and worldwide impact Paris, France. 10-13 September 2008

Published: 23 September 2008

BMC Proceedings 2008, 2(Suppl I):SIO

This abstract is available from: http://www.biomedcentral.com/I753-656I/2/SI/SI0

(c) 2008 Drögemüller et al; licensee BioMed Central Ltd.

Toxoplasma (T.) gondii infects astrocytes, neurons and microglia cells in the CNS and, after acute encephalitis, persists within neurons. Robust astrocyte activation is a hallmark of Toxoplasma encephalitis (TE); however, the in vivo function of astrocytes is largely unknown. To study their role in TE, we generated C57BL/6 GFAP-Cre gp $130 \mathrm{fl} /$ $\mathrm{fl}$ mice, which lack gp130, the signal transducing receptor for IL-6 family cytokines, in their astrocytes. In TE of wildtype mice, the gp130 ligands IL-6, IL-11, IL-27, LIF, oncostatin $\mathrm{M}$, ciliary neurotrophic factor, B cell stimulating factor, and cardiotrophin-1 were upregulated. In addition, $\mathrm{GFAP}^{+}$astrocytes of gp $130 \mathrm{fl} / \mathrm{fl}$ control mice were activated, increased in number, and efficiently restricted inflammatory lesions and parasites, thereby, contributing to survival from TE. In contrast, $T$. gondii-infected GFAPCre gp $130^{\mathrm{fl} / \mathrm{fl}}$ mice lost $\mathrm{GFAP}^{+}$astrocytes in inflammatory lesions resulting in an inefficient containment of inflammatory lesions, impaired parasite control and, ultimately, a lethal necrotizing TE. Production of IFN-gamma and IGTP, which mediate parasite control in astrocytes, were even increased in GFAP-Cre gp $130^{\mathrm{fl} / \mathrm{fl}}$ mice indicating that instead of the direct anti-parasitic effect the immunoregulatory function of GFAP-Cre gp $130^{\mathrm{fl} / \mathrm{fl}}$ astrocytes was disturbed. Correspondingly, in vitro infected GFAP-Cre gp $130^{\mathrm{fl} / \mathrm{fl}}$ astrocytes inhibited growth of $T$. gondii effi- ciently after stimulation with IFN-gamma, whereas neighbouring non-infected and TNF-stimulated GFAP-Cre gp130 fl/fl astrocytes became apoptotic. Collectively, these are the first experiments demonstrating a crucial function of astrocytes in CNS infection. 